\title{
ANÁLISE DO CONTEÚDO ESTOMACAL DE Moenkhausia intermedia (Eigenmann, 1908) (CHARACIFORMES: CHARACIDAE), PROVENIENTE DA LAGOA DO DIOGO, BACIA DO RIO MOGI- GUAÇU, LUÍS ANTÔNIO, ESTADO DE SÃO PAULO
}

\author{
MACHADO, Clycie Aparecida da Silva ${ }^{1}$ \\ RODRIGUES, Thales ${ }^{1}$ \\ MORALES, Adriana Coletto ${ }^{2}$
}

Recebido em: 2009-05-04
Aprovado em: 2009-08-07

ISSUE DOI: $10.3738 / 1982.2278 .200$

\begin{abstract}
RESUMO: O presente trabalho teve como objetivo analisar a dieta de Moenkhausia intermedia (Eigenmann, 1908), provenientes da Lagoa do Diogo, bacia do rio Mogi-Guaçu, município de Luís Antônio, São Paulo. Foram examinados 16 estômagos através do método de frequiência de ocorrência $(\% \mathrm{FO})$. A análise mostrou que $28,79 \%$ dos itens alimentares são autóctones, $48,48 \%$ alóctones e $22,73 \%$ de origem não identificada. Os resultados indicam que $M$. intermedia pertence a guilda alimentar onívora, com tendência à insetivoria. Os itens alóctones foram os mais freqüentes, sendo que as sementes perfizeram $100 \%$ de freqüência de ocorrência e os insetos terrestres $68,75 \%$. Por isso, se considera de grande importância fontes alóctones de alimento, sendo necessária a vegetação marginal íntegra como fonte de recurso alimentar para a maioria dos invertebrados aquáticos e peixes.
\end{abstract}

Palavras-chave: Moenkhausia intermedia. Lagoa do Diogo. Bacia do rio Mogi-Guaçu. Frequiência de ocorrência. Conteúdo estomacal.

\section{ANALYSIS OF STOMACH CONTENTS OF Moenkhausia intermedia (Eigenmann, 1908) (Osteichthyes: Characiformes), from the Lake DIOGO, LUÍS ANTÔNIO - SP}

SUMMARY: The goal of this study was to analyze the diet of Moenkhausia intermedia (Eigenmann, 1908) from Diogo Lake, Rio Mogi-Guaçu basin, municipality of Luís Antônio, State of São Paulo, southeastern Brazil. Sixteen stomachs were examined using the method of frequency of occurrence (\%FO). According to our analysis $28.79 \%$ of the food items were autochthonous, $48.48 \%$ allochthonous and $22.73 \%$ from non identified origins. Our results indicate that $\mathrm{M}$. intermedia belongs to the omnivorous food guild, with a tendency to insectivory. The allochthonous items were the most frequent, with seeds showing a $100 \%$ frequency of occurrence and terrestrial insects $68.75 \%$. Hence, an intact riparian vegetation is considered of great importance as a source of food for most aquatic invertebrates and fish.

Keywords: Moenkhausia intermedia. Diogo Lake. Rio Mogi-Guaçu basin. Frequency of occurrence. Stomach contents.

\section{INTRODUÇÃO}

São conhecidas atualmente em torno de 515 famílias e 27.977 espécies de peixes no planeta, das quais aproximadamente um terço (11.952 espécies) ocupam águas doces

\footnotetext{
${ }^{1}$ Graduados em Ciências Biológicas pela FE/FFCL

${ }^{2}$ Dr $^{\mathrm{a}}$. em Ciências Biológicas - Área Biologia Comparada. Prof ${ }^{\mathrm{a}}$. FE/FFCL
} 
permanentes (NELSON, 2006). A América do Sul contém a mais rica ictiofauna de água doce do mundo, porém a avaliação e compreensão dessa rica diversidade são negativamente afetadas pelo conhecimento incompleto de sua ecologia, biologia e sistemática (MENEZES, 1996). Em termos de diversidade, estima-se que o número final de espécies de água doce neotropicais chegaria a 5.000 (BÖHLKE et al, 1978). Segundo Schaefer (1998) a estimativa é em torno de 8.000 espécies, o que, proporcionalmente, representa um oitavo de toda a biodiversidade estimada de vertebrados viventes (VARI; MALABARBA, 1998).

No Brasil, a fauna de peixes de água doce contêm aproximadamente 39 famílias, 517 gêneros válidos e mais de 2.500 espécies (BUCKUP et al., 2007), esta diversidade ainda não está bem compreendida, e estima-se que existam aproximadamente 35\% a 40\% das espécies de peixes por serem descritas (SCHAEFFER, 1998; REIS et al, 2003; BUCKUP et al., 2007).

No Estado de São Paulo são registradas 22 famílias e aproximadamente 160 espécies de peixes (CASTRO; MENEZES, 1998). A maior parte dessa fauna é composta por espécies de peixes de pequeno porte (menos de $15 \mathrm{~cm}$ de comprimento) que habitam pequenos riachos de cabeceiras, apresentando elevado grau de endemismo, distribuição geográfica restrita, sem valor comercial e dependentes da vegetação ripária para alimentação, reprodução e abrigo (CASTRO; MENEZES, 1998). Tais comunidades têm sido fortemente afetadas pela construção de barragens; uso descontrolado de pesticidas e fertilizantes; destruição de florestas, principalmente da vegetação ripária; assoreamentos e introdução de espécies de outras bacias hidrográficas (BÖHLKE et al., 1978; MENEZES, 1996; CASTRO; MENEZES, 1998).

Os ambientes lênticos são locais de águas quase paradas ou lentamente renovadas (lagos, lagoas, represas, reservatórios). Em geral, apresentam padrões verticais e horizontais das variáveis físicas, químicas e biológicas que influenciam diretamente a composição, a estrutura e a dinâmica da assembléia de peixes presentes no sistema. (OLIVEIRA; GOULART, 2000). Os peixes usam a estrutura física do ambiente, tais como: rochas, madeiras submersas, macrófitas aquáticas como abrigo contra predadores e como sítios de forrageamento e reprodução, como também as próprias macrófitas podem ser utilizadas como hábitats pelos peixes.

Lowe-McConnel (1987) postula que, em geral, peixes de regiões tropicais não apresentam especializações tróficas, modificam sua dieta conforme o crescimento, mudanças de biótopo ou de acordo com flutuações estacionais. Esta sazonalidade é induzida principalmente por mudanças no nível d'água que afetam o habitat e conseqüentemente a disponibilidade de alimento (WINEMILLER; JEPSEN, 1998; ARANHA et al., 2000). Mesmo considerando que a ação desestabilizadora de fatores físicos do ambiente passa a 
manter as populações abaixo da capacidade de suporte do meio, diminuindo a competição por alimento (WIENS, 1987). Ainda assim, alguns autores acreditam que a partilha de recursos nestes ambientes é um importante fator ecológico para a redução da competição entre as espécies, permitindo sua coexistência (ARANHA et al., 1998).

Espécies de peixe de pequeno porte correspondem a aproximadamente $50 \%$ do total de espécies de peixes de água doce descritas da América do Sul e mostram em grau elevado de endemismo. O estudo da sistemática, evolução e biologia geral das espécies de peixe de pequeno porte são, sem dúvida, o grande desafio da ictiologia sul-americana no início deste século (CASTRO; MENEZES, 1998; CASTRO, 1999).

A espécie Moenkhausia intermedia (Characiformes: Characide) (Figura 1), é considerada de pequeno porte com até $8 \mathrm{~cm}$ de comprimento (FISHBASE, 2008) e um importante componente da cadeia alimentar, especialmente para outras espécies de peixes, de aves e de outros carnívoros que vivem na planície de inundação (LIZAMA; AMBRÓSIO, 2003). É conhecida como viuvinha no Estado de São Paulo e como pequi, piqui na planície de inundação do Alto rio Paraná (LIZAMA; AMBRÓSIO, 2003). É distribuída no rio Amazonas, rio Paraguai, rio Tietê, Bolívia e Guianas (FOWLER, 1948). Trata-se de uma espécie forrageira, considerada onívora e que pode ser encontrada em ambientes lênticos ou em lóticos (ESTEVES; GALETTI, 1994; LIZAMA; AMBRÓSIO, 1997)

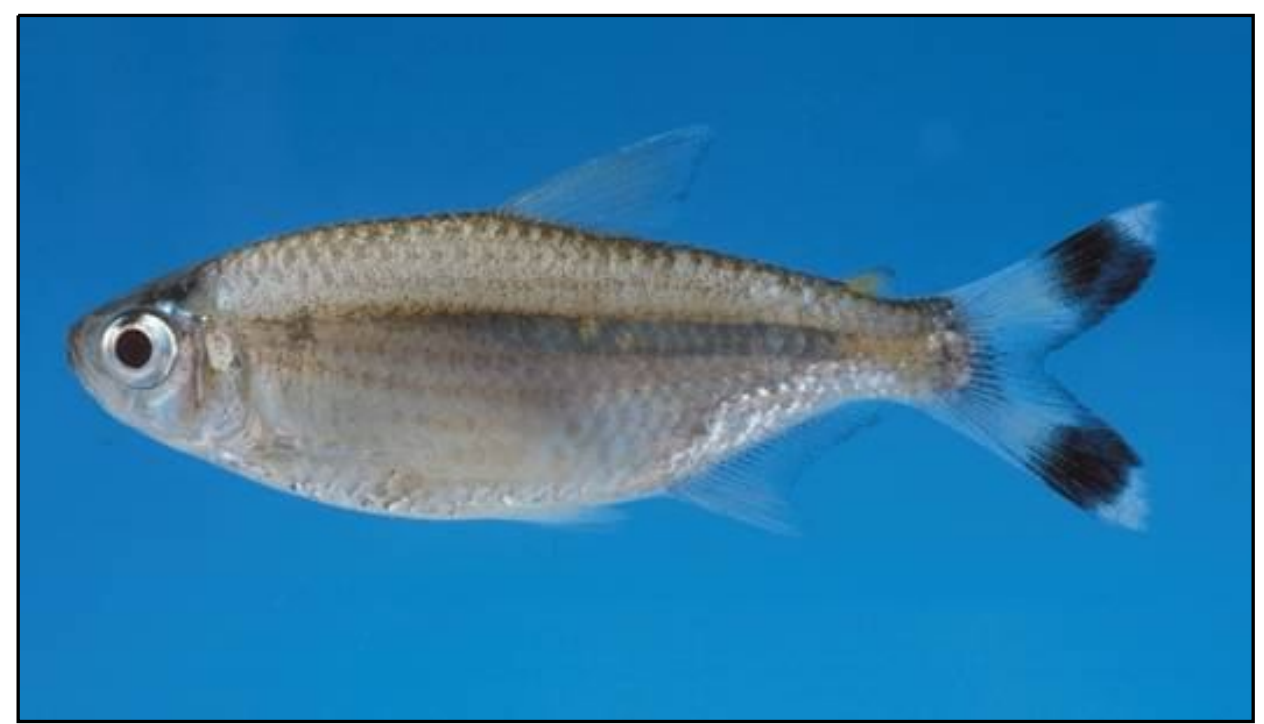

FIGURA 1. Moenkhausia intermedia (EIGENMANN, 1908). Foto: R. M. C. Castro

A Lagoa do Diogo (Figura 2) é uma lagoa marginal da planície de inundação do rio Mogi-Guaçu, situada na unidade de conservação ambiental Estação Ecológica de Jataí (EEJ)

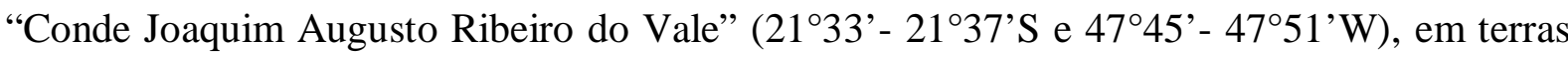


do domínio da Fazenda Pública do Estado, no município de Luís Antônio, região centro-leste do Estado de São Paulo (Figura 3) (TANIGUCHI et al., 2004).

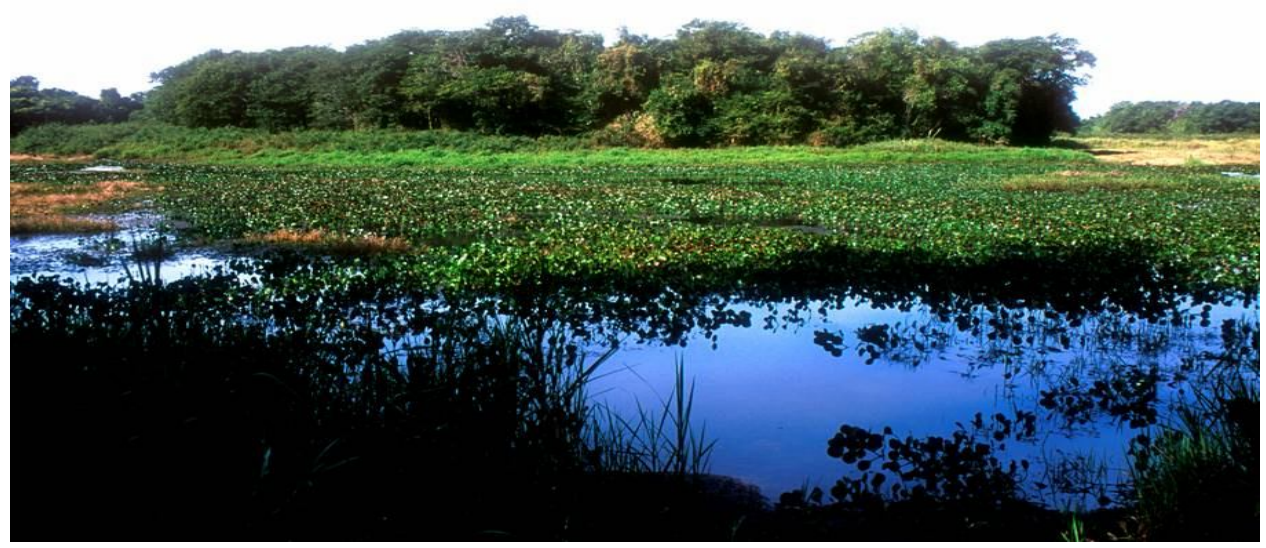

FIGURA 2. Lagoa do Diogo, Estação Ecológica de Jataí, Luís Antônio, SP. Foto: R. M. C. Castro.

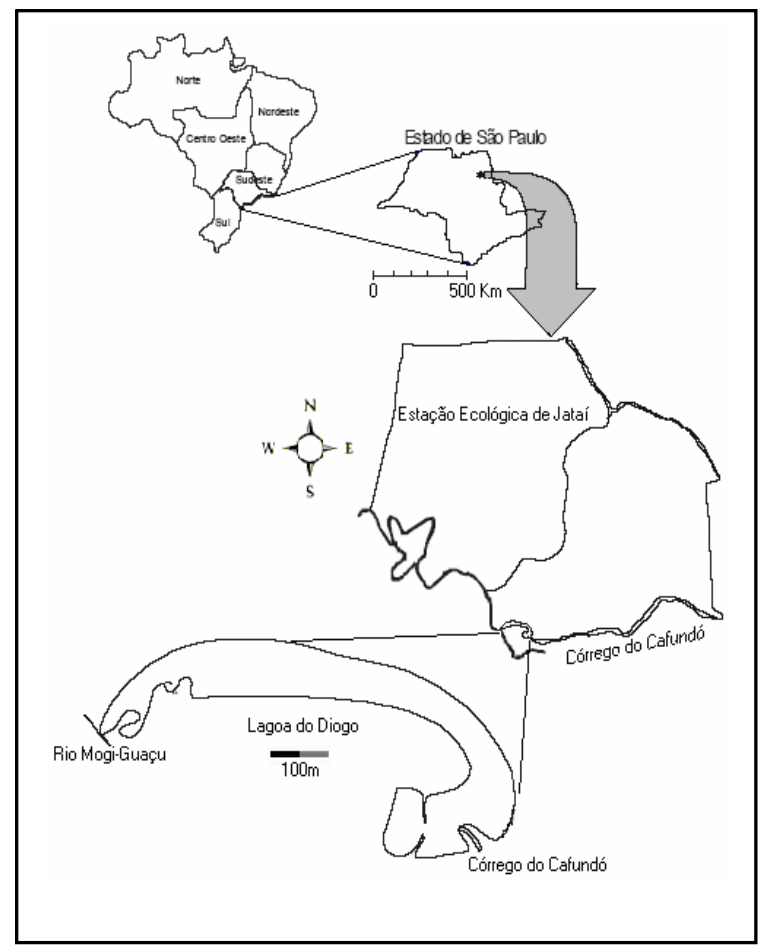

FIGURA 3. Localização da Lagoa do Diogo na Estação Ecológica de Jataí em Luís Antônio, SP. Fonte: Taniguchi et al (2004).

A lagoa do Diogo é um sistema de drenagem e possui uma conexão direta com o canal principal do rio Mogi-Guaçu durante o ano todo, além de receber influxo permanente do córrego do Cafundó em sua porção leste (TANIGUCHI et al., 2004). É uma das maiores lagoas marginais do rio Mogi-Guaçu, dentro da referida área de conservação ambiental (MAGRIN; SENNA, 2000). 


\section{BIOLOGIA ALIMENTAR}

A alimentação é um dos fatores essenciais na vida de todos os seres vivos, seja para repor ou acrescentar elementos necessários utilizados no crescimento de órgãos e tecidos do organismo (ZAVALA-CAMIN, 1996). O desenvolvimento de uma população depende da disponibilidade de alimento, do encontro entre presa e predador, bem como uma adequada transferência de energia entre os níveis tróficos (KREBS, 1998). Dessa forma estudos sobre a biologia alimentar são de grande importância para a compreensão da organização trófica geral dos ecossistemas e o conhecimento quantitativo dos mecanismos biológicos de interações entre espécies, tais como predação e competição (HERRÁN, 1998).

O conhecimento dos hábitos alimentares são necessários para o entendimento da amplitude de uso de recursos ao longo da distribuição temporal e espacial de qualquer espécie (KREBS, 1998), e da dinâmica das populações e comunidades (CUNNIGHAM, 1989).

$\mathrm{O}$ estudo da dieta de espécies e da interação alimentar destas com o meio fornece importantes informações ecológicas, bem como sobre o comportamento de peixes diante de variações nas condições ambientais e do alimento disponível, fornecendo ainda subsídios para a compreensão de mecanismos que permitem a coexistência e a exploração dos recursos de um mesmo sistema por várias espécies (GOULDING, 1980).

Variações na dieta podem estar relacionadas à disponibilidade de alimento ao longo do ano, seleção ativa de alimentos preferidos de acordo com a escolha individual, mudanças ontogenéticas ou à presença de outras espécies (LOWE-McCONNELL, 1987). A sazonalidade na oferta alimentar é um importante fator que pode provocar variações na dieta dos peixes e, conseqüentemente, na amplitude do nicho alimentar das espécies. A medida da amplitude do nicho alimentar de uma espécie possibilita uma avaliação quantitativa da maior ou menor especialização dos organismos em utilizar recursos do ambiente (KREBS, 1989).

O presente trabalho teve como objetivo caracterizar a dieta de Moenkhausia intermedia, utilizando 16 espécimes provenientes da Lagoa do Diogo, cidade de Luís Antônio, região centro-leste do Estado de São Paulo. Todos os exemplares examinados estavam depositados no Laboratório de Ictiologia de Ribeirão Preto, SP (LIRP - USP), no lote $\mathrm{n}^{\circ} .5095$ e foram emprestados para a realização deste trabalho. 


\section{MATERIAL E MÉTODO}

A coleta foi realizada no dia 08 de outubro de 2004, pela equipe do LIRP - Laboratório de Ictiologia de Ribeirão Preto - USP, na Lagoa do Diogo, dentro da Estação Ecológica de Jataí no município de Luís Antônio, SP, Brasil. Para a captura dos peixes foi utilizada uma rede de arrasto com $20 \mathrm{~m}$ de comprimento, contendo 350 malhas de $5 \mathrm{~mm}$ entre nós, o que corresponde a aproximadamente $1.7 \mathrm{~m}$ de altura. Os exemplares coletados foram fixados em formol $10 \%$, posteriormente separados por semelhança morfológica externa, identificados até o nível de espécie, em seguida transferidos para álcool 70\% para sua conservação, rotulados e tombados na coleção do LIRP - USP.

A análise do conteúdo estomacal dos exemplares foi efetuada nos laboratórios didáticos nos campus I e II da Fundação Educacional de Ituverava - FFCL e FAFRAM, Ituverava, SP, no período de setembro a outubro de 2007.

Os espécimes utilizados sofreram uma incisão entre a cloaca e a nadadeira peitoral, em seguida o estômago foi retirado e posteriormente depositado em tubos plásticos com álcool a $70 \%$, devidamente etiquetado. Para cada estômago examinou-se os itens alimentares com o auxílio de uma lupa (aumento de 2 x 10) e o material foi identificado através das chaves de identificação de Bicudo; Bicudo (1970) e Merritt; Cumminsi (1996).

Após a identificação dos itens alimentares foi utilizado o método de frequiência de ocorrência (\% FO) para a descrição quantitativa da dieta. Este método é o mais usual para análises de conteúdos estomacais (ARANHA, 1993). Os itens alimentares foram classificados em autóctones (itens de origem aquática), alóctones (itens de origem terrestre, ou seja, material orgânico, como vegetais, insetos, que entram no sistema aquático), e origem indeterminada (fragmentos de vegetais, fragmentos de insetos e detritos).

\subsection{DESCRIÇÃO QUANTITATIVA DA DIETA}

Há três métodos que descrevem quantitativamente a dieta de um organismo, são eles: freqüência de ocorrência, composição percentual do número e composição percentual por peso do alimento, onde cada método providencia distintas informações a respeito da dieta.

Entretanto a frequiência de ocorrência é o método mais eficiente para a análise quantitativa da dieta de peixes. Com base na análise do conteúdo estomacal de todos os espécimes, uma lista é elaborada com os vários tipos de alimento e a seguir registra-se a presença ou ausência deste item alimentar em cada espécime analisado. Quando todos os 
espécimes forem examinados, a proporção dos peixes que contêm um ou mais de um dado tipo de alimento é calculado como a frequiência de ocorrência por aquele tipo de alimento. Assim, se 18 peixes de um total de 22 contêm um determinado item, a freqüência de ocorrência deste item na dieta será de 82\% (BOWEM, 1989).

Estes resultados indicam quais peixes na amostra funcionam como uma simples unidade alimentar. Se quase todos os peixes contêm em seu estômago o mesmo tipo de presa, isto será claramente documentado com a uniformidade de uma alta freqüência de ocorrência. Mas, se um único peixe concentra sua alimentação em um só tipo de comida, isto será refletido em baixa freqüência de ocorrência deste item. Se um grupo de peixes onívoros, que compartilham diversos tipos de algas filamentosas, mas são especializados em um só tipo de invertebrado, terão alta freqüência de ocorrência para a maioria dos tipos de algas e baixos valores para este tipo de invertebrado (BOWEM, 1989).

Uma alta frequiência de ocorrência não significa que um dado tipo de alimento é de importância nutricional para o consumo, até porque este item pode ser consumido com grande regularidade, mas em pequena quantidade. Os dados de freqüência de ocorrência, descrevem a uniformidade com que cada grupo de peixes seleciona sua dieta, mas não indica a importância dos vários tipos de alimentos selecionados (BOWEM, 1989).

\section{RESULTADO}

A análise dos 16 estômagos de Moenkhausia intermedia através do método de freqüência de ocorrência (\%FO) mostrou que 28,79\% dos itens alimentares são autóctones, $48,48 \%$ alóctones e $22,73 \%$ de origem indeterminada. Todos os indivíduos possuíam conteúdo estomacal. No total, as sementes perfizeram $100 \%$ de ocorrência, insetos terrestres $68,75 \%$, insetos aquáticos $56,25 \%$, detritos $43,75 \%$, fragmentos de insetos $37,5 \%$, escamas $12,5 \%$, algas $12,5 \%$, fragmentos de vegetais $12,5 \%$, ovos $6,25 \%$ e microcrustáceos (Cladocera) 6,25\% de ocorrência. O item que obteve maior \%FO foram as sementes presentes em todos os estômagos (Tabela 1). A tabela 2 apresenta os dados da dieta alimentar presentes no estômago de cada indivíduo analisado, reconstituindo portanto a informação alimentar.

O item "detritos" são fragmentos originários de animais ou vegetais em decomposição. O item "fragmentos de insetos" foi representado por restos de pernas, pedaços de asas e fragmentos de exoesqueleto, não sendo possível sua identificação a um nível taxonômico. 
Com base nos itens alimentares, M. intermedia foi considerada uma espécie onívora com tendência à carnivoria. Usa-se o termo onívoro para referir-se às espécies que utilizam alimentos pertencentes a dois ou mais níveis tróficos (VADAS, 1990). Quando há um domínio de algum dos itens, refere-se às espécies como: onívoro com tendência à carnivoria ou onívoro com tendência à herbivoria (ANDRIAN et al., 1994).

TABELA 1. Análise dos itens alimentares de $M$. intermedia provenientes da Lagoa do Diogo, Luís Antônio, SP e freqüência de ocorrência dos itens examinados.

Moenkhausia intermedia $(\mathrm{N}=16 ; \mathrm{Cp}=4,2-6,0 \mathrm{~cm} ; \mathrm{H}=1,46 \mathrm{~cm} ; \mathrm{Nvaz}=0)$

\section{Itens autóctones}

Algas

Chironomidae

Cladocera

Culicidae

Diptera

Escamas

Odonata

Ovos

Trichoptera genera

\section{Oc}

2

1

1

4

5

2

1

1

2

3

3

2

2

1

1

3

16

1
$\%$ FO

12,5

6,25

6,25

25

31,25

12,5

6,25

6,25

12,5

18,75

18,75

12,5

12,5

6,25

6,25

18,75

100

6,25

\section{Origem indeterminada}

Fragmentos de insetos

6

37,5

Fragmentos de vegetais

2

12,5

Detritos

Total 66

Número de exemplares examinados $(\boldsymbol{N})$, amplitude de comprimento padrão $(\boldsymbol{C p})$, altura média $(\boldsymbol{H})$, número de estômagos vazios (Nvaz), total de estômagos em que foi encontrado o item $(\boldsymbol{O C})$, freqüência de ocorrência $(\boldsymbol{\%} \mathbf{F} \mathbf{O})$. 
TABELA 2. Lista da dieta presente no estômago de cada espécime de Moenkhausia intermedia.

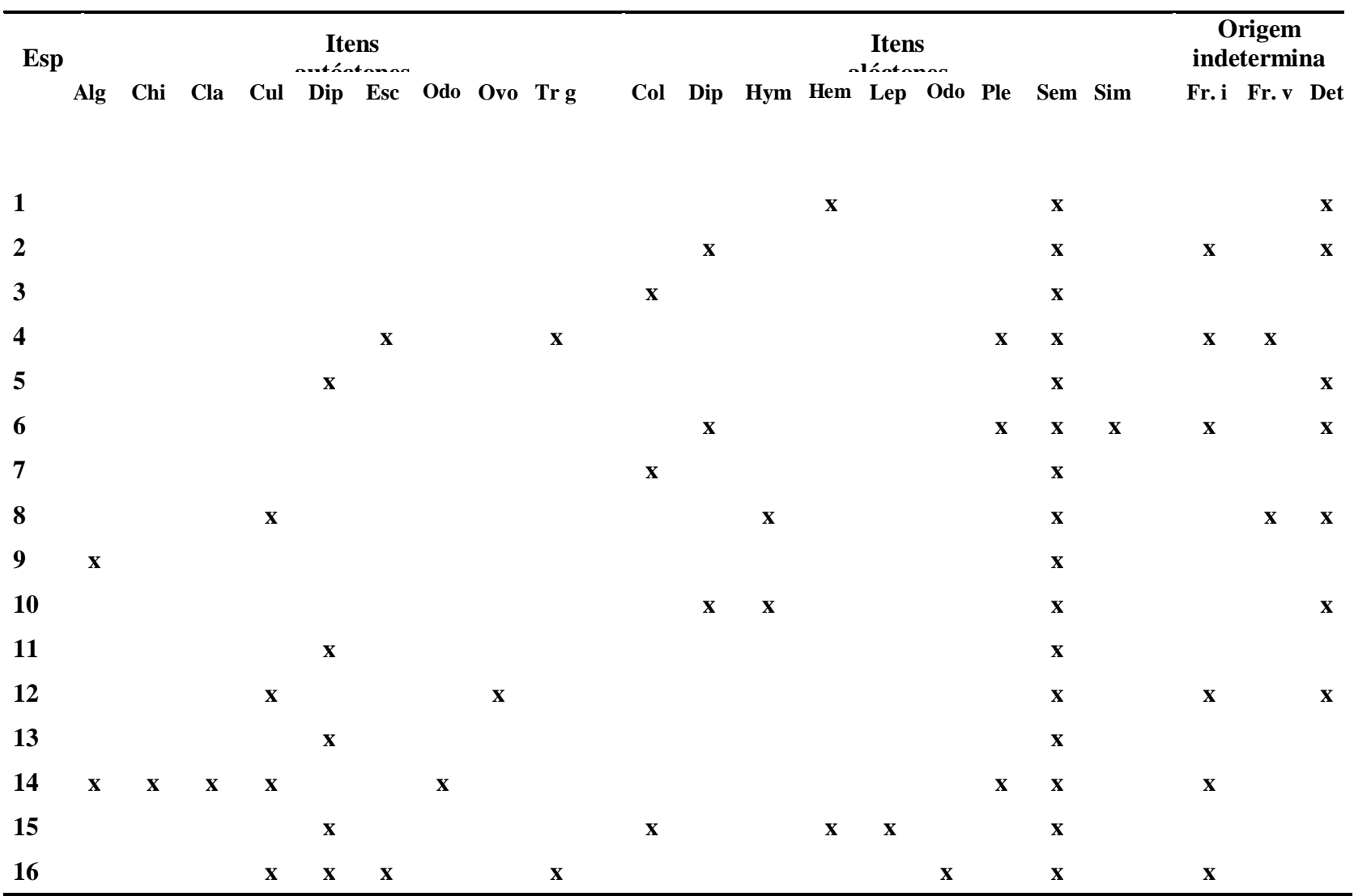

Espécimes (Esp), algas (Alg), chironomidae (Chi), cladocera (Cla), culicidae (Cul), diptera (Dip), escamas (Esc), odonata (Odo), ovos (Ovo), trichoptera genera (Tr g), coleoptera (Col), hymenoptera (Hym), hemiptera (Hem), lepidoptera (Lep), plecoptera (Ple), sementes (Sem), simuliidae (Sim), fragmentos de insetos (Fr. i), fragmentos de vegetais (Fr. v), detritos (Det).

\section{DISCUSSÃO}

Espécies de peixes onívoras possuem grande variação morfológica, o que provavelmente se relaciona à falta de especializações que caracterizam membros deste grupo trófico (HORN, 1998). Os onívoros aproveitam grande variedade de alimentos disponíveis em diversos locais, por esse motivo uma mesma espécie pode apresentar dieta diversificada, dependendo da região e da época do ano (ZAVALA-CAMIN, 1996).

Ao comparar dados da dieta encontrados em Moenkhausia intermedia, proveniente da Lagoa do Diogo com os dados obtidos em outros trabalhos, observa-se que os resultados corroboram. Esta espécie foi considerada onívora em lagoas do rio Mogi-Guaçu (ESTEVES; GALETTI, 1994). Como também na Lagoa do Diogo, Luís Antônio, SP, onde, 77 \% dos exemplares analisados foram coletados durante a estação chuvosa, neste período sua alimentação apresentou-se bastante diversificada, constituída principalmente por escamas, tecido vegetal, algas filamentosas e insetos aquáticos, já na estação seca, houve aumento considerável de tecido vegetal, algas filamentosas e insetos terrestres na dieta desta espécie 
(MESCHIATTI, 1995). Também foi considerada onívora em um estudo realizado no reservatório de Capivara, município de Porecatu, localizado no médio Paranapanema, entre os Estados do Paraná e São Paulo, onde se identificou principalmente detrito, restos de vegetais em menor quantidade, Ostracoda e Copepoda (TEIXEIRA; BENNEMANN, 2007).

O estudo da alimentação de espécies forrageiras em uma lagoa marginal do rio MogiGuaçu, SP, verificou que a dieta de $M$. intermedia foi onívora e sua atividade alimentar ao longo do ano mostrou-se variável, apresentando relações com a disponibilidade alimentar, que aparentemente foi maior nos períodos de fevereiro-março, correspondente ao pico da cheia, e setembro-outubro, coincidente com o reinício das chuvas. Estes períodos determinaram uma mudança quantitativa e qualitativa nos itens consumidos (ESTEVES, 1992).

Entretanto, não é em todos os estudos sobre alimentação envolvendo esta espécie, que os resultados indicaram-na como onívora. Isso pode ocorrer devido às diferenças na disponibilidade de alimento no ambiente em que essas espécies foram estudadas, e também quanto ao número de exemplares examinados (UIEDA, 1983).

Agostinho et al., (1997) e Hahn et al., (2003), caracterizaram-na como insetívora na planície do rio Paraná, e Gaspar da Luz et al, (2001) registrou-a como zooplanctófaga na lagoa da Pousada, Ilha Mutum, alto rio Paraná, Porto Rico, PR. Tendem a freqüentar regiões pelágicas em cardumes, onde se alimentam de zooplâncton, como o observado no rio Tibagi (BENNEMANN et al, 2000, BENNEMANN; SHIBATTA, 2002).

Cabe ressaltar que Moenkhausia sanctaefilomenae (STEINDACHNER, 1907), outra espécie do mesmo gênero, foi considerada insetívora em um estudo sobre a alimentação dos peixes em um riacho do parque estadual Morro do Diabo, bacia do Alto rio Paraná (CASATTI, 2002).

\section{CONCLUSÃO}

Através dos resultados obtidos, conclui-se que Moenkhausia intermedia, possui o hábito alimentar onívoro com tendência à carnivoria, devido a variedade alimentar encontrada. Os itens de origem alóctone foram os mais freqüentes na dieta, representados principalmente por sementes e insetos terrestres, por isso se considera de grande importância fontes alóctones de alimento para a ictiofauna que, em muitos locais estão sendo devastadas.

A vegetação ripária, a serapilheira, os insetos terrestres e todos os outros itens alimentares de origem alóctone são importantes, pois são carreados para o corpo d'água, e servem de substrato energético para a maioria dos invertebrados aquáticos e peixes. Deste 
modo, alterações na estrutura e composição da vegetação ripária provavelmente implicarão em impactos negativos na integridade do ambiente aquático, refletindo diretamente na qualidade de vida e da alimentação dos organismos que aí vivem. Parte desta conclusão também é compartilhada por Ferreira (2002), na realização do estudo de peixes de um riacho da bacia do rio Mogi-Guaçu.

\section{AGRADECIMENTOS}

Agradecemos ao Laboratório de Ictiologia de Ribeirão Preto (LIRP), do Departamento de Biologia da FFFCLRP - Universidade de São Paulo pela permissão de utilização do material de M. intermédia analisado por nós. E em especial ao Prof. Dr. Ricardo M. C. Castro e ao Dr. Hertz F. dos Santos (ambos do LIRP) pela permissão de uso dos dados ambientais, fotos e apoio na confecção deste trabalho.

\section{REFERÊNCIAS}

AGOStinho, A. A. et al. Estrutura trófica. In: VAZZOLER, A. E. A. M. et al. (Org.). A planície de inundação do alto rio Paraná: aspectos físicos, biológicos e socioeconômicos. Maringá: EDUEM, 1997. p. 229-248.

ANDIAN, I. F. et al. Espectro alimentar e similaridade na composição da dieta de quatro espécies de Leporinus (Characiforme, Anostomidae) do rio Paraná (2210' - 2250'S / 531’- 5340’W), Brasil. Revista UNIMAR, v.16, supl. 3, p. 97-106, 1994.

ARANHA, J. M. R. Método para análise quantitativa de algas e outros itens microscópios de alimentação de peixes. Acta Biol. Par, Curitiba, (1, 2, 3, 4), p. 71-76, 1993.

ARANHA, J. M. R.; TAKEUTI, D. F.; YOSHIMURA, T. Habitat use and food partitioning of the fishes in the Mergulhão stream (coastal stream of Atlantic Forest, Brazil). Revista de Biologia Tropical, San José, v.46, n.4, p. 951-959, 1998.

ARANHA, J. M. R.; GOMES, J. H. C.; FOGAÇA, E. N. O. Feeding of two species of Characidium, $C$. lanei and $C$. pterostictum (Characidiinae) in a coastal stream of Atlantic Forest (Southern Brazil). Brazilian Archives of Biology and Technology, Curitiba, v.43, n.5, p. 527-531, 2000.

BENNEMANN, S. T.; SHIBATTA, O. A. Dinâmica de uma assembléia de peixes do rio Tibagi. In: MEDRI, M. E. et al (Orgs.). A bacia do rio Tibagi. Londrina: UEL, 2002. p. 433442.

Bennemann, S. T.; ShIBATTA, O. A.; GARAVELlo, J. C. Peixes do rio Tibagi: uma abordagem ecológica. Londrina: EDUEL, 2000. 62p. 
BICUDO, C. E. M.; BICUDO, R. M. T. Algas de águas continentais brasileiras: chave ilustrada para identificação de gêneros. São Paulo: Fundação Brasileira para o desenvolvimento do ensino de ciências, 1970. 228p.

BOWEM, S. H. Quantitative description of the diet. In: Nielsen, L. A.; Johnson, D. L. Fisheries Techniques. American Fisheries Society. Bethesda, Maryland, 1989. 468p.

BÖHLKE, J.; WEITZMAN, S. H.; MENEZES, N. A. Estado atual da Sistemática de peixes de água doce da América do Sul. Acta Amazônica, v.8, n.4, p. 657-677, 1978.

BUCKUP, P. A., N. A. MENEZES \& M. S. GHAZZI (eds.), 2007. Catálogo das espécies de peixes de água doce do Brasil. Série livros 23, Museu Nacional, Rio de Janeiro, 195 p.

CASATI, L. Alimentação dos peixes em um riacho do Parque Estadual Morro do Diabo, Bacia do Alto rio Paraná, sudeste do Brasil. São José do Rio Preto: Universidade Estadual Paulista, 2002.

CASTRO, R. M. C.; MENEZES, N. A. Estudo diagnóstico da diversidade de peixes do Estado de São Paulo. In: CASTRO, R. M. C.; JOLY, C. A.; BICUDO, C. E. M. (Org.). Biodiversidade do Estado de São Paulo, Brasil: síntese do conhecimento ao final do século XX: vertebrados. v.6. São Paulo: WinnerGraph - FAPESP, 1998. p. 1-13.

CASTRO, R. M. C. Evolução da ictiofauna de riachos sul-americanos: padrões gerais e possíveis processos causais. In: CARAMASCHI, E. P.; MAZZONI, R.; PERES-NETO, P. R. (Orgs.). Ecologia de peixes de riachos. v.6. Rio de Janeiro: PPGE-UFRJ, 1999. p. 139-155. (Série Oecologia Brasiliensis).

CUNNINGHAM, P. T. M. Observações sobre o espectro alimentar de Ctenosciaena gracilirrhus (Metzellar), Scianidae. Rev. Brasil. Biol. v.49, n.2, p. 335-339, 1989.

EIGENMANN, C. H. Preliminary descriptions of new genera and species of tetragonopterid characins. (Zoölogical Results of the Thayer Brazilian expedition.). Bulletin of the Museum of Comparative Zoology, v. 52, n.6, p. 91-106, 1908.

ESTEVES, K. E. Alimentação de cinco espécies forrageiras (Pisces, Characidae) em uma lagoa marginal do rio Mogi-Guacu, SP. São Carlos: UFSCar, 1992. 230p. Tese de Doutorado (Ecologia)-UFSCar. CCBS-Centro de Ciências Biológicas e da Saúde. ERN.

ESTEVES, K. E.; GALETTI, P. M. Feeding ecology of Moenkhausia intermedia (Pisces, Characidae) in a small oxbow lake of Mogi-Guaçu River, São Paulo, Brazil. Verh. Internat. Verein. Limnol. v.25, p. 2198-2204. 1994.

FERREIRA, K. M. Aspectos da biologia e ecomorfologia dos peixes de um riacho da bacia do rio Mogi-Guaçu, SP. 2002. 148p. Dissertação (Mestrado). Universidade de São Paulo.

FISHB ASE. Banco de dados. Disponível em:

$<$ http://www.fishbase.org/Summary/speciesSummary.php?ID=26474\&genusname=Moenkha usia\&speciesname=intermedia>. Acesso em: 03 de junho de 2008.

FOWLER, H. W. Os peixes de água doce do Brasil 1. Arqu. Zool. Estado de S. Paulo, v.6, n.1, 204 p. 1948. 
GASPAR da LUZ, K. D. da et al. Caracterização trófica da ictiofauna de três lagoas da planície aluvial do alto rio Paraná, Brasil. Acta Scientiarum. Maringá, v.23, n.2, p. 401-407, 2001.

GOULDING, M. The fishes and the Forest: Explotations in Amazonian Natural History. Berkeley: University of California Press, 1980, 280p.

HAHN N. S. et al. Estrutura trófica da ictiofauna da planície de inundação do alto rio Paraná Nupélia/PEA. Maringá: Universidade Estadual de Maringá, 2003. p. 123-126.

HAHN, N. S.; DELARIVA, R. L. Métodos para avaliação da alimentação natural de peixes: o que estamos usando? Interciencia, v.28, n.2, p. 100-104, 2003.

HERRÁN, R. A. Analisis de contenidos estomacales em peces. Revision bibliografica de los objetivos y la Oceanografia, n.63, p. 1-73, 1998.

HORN, M. H. Feeding and digestion. In: EVANS, D. H. The physiology of the fishes. 2.ed. Boca Raton: CRC Press, 1998. p. 43-64.

KÖPPEN, W. Das Geographische System der Klimatologic. Berlin: 2.ed., 1936. 44p.

KREBS, C. J. Ecological Methodology. 2.ed. S.L.: Harper and Collins Publishers, 1998. $581 \mathrm{p}$.

KREBS, C. J. Ecological Methodology. New York, Harper and Row Publishers, 1989. xii + 700p.

LIZAMA, M. L. A. P.; AMBRÓSIO, A. M. Relação peso-comprimento em espécies forrageiras na planície de inundação do alto rio Paraná. In: ENCONTRO BRASILEIRO DE ICTIOLOGIA, 12, Anais. 1997, São Paulo.

LIZAMA, M. L. A. P.; AMBRÓSIO, A. M. Crescimento, recrutamento e mortalidade do pequi, Moenkhausia intermedia (Osteichthyes, Characidae) na planície de inundação do alto rio Paraná, Brasil. Acta Scientiarum: biological sciences. Maringá, v.25, n.2, p. 329-333, 2003.

LOWE-McCONNELL, R. H. Ecological studies in tropical fish Communities. Cambridge: Cambridge University Press, 1987. 382p.

MAGRIN, A. G. E.; SENNA, P. A. C. Diatomáceas (Bacillariopphyta) da Lagoa do Diogo e seus trechos fluviais: Córrego Cafundó e rio Mogi-Guaçu .1. Classes Coscinodiscophyceae e Fragilariophyceae (Fragilariaceae). In: SANTOS, J. E.; PIRES, J. S. R. Estação ecológica de Jataí. São Carlos: Rima, 2000. v. 2, p. 403-413.

MESCHIATTI, A. J. Alimentação da comunidade de peixes de uma lagoa marginal do rio Mogi-Guaçu, SP. Acta Limnologica Brasiliensia, v.8, p. 115-137, 1995.

MENEZES, N. A. Methods for assessing freshwater fish diversity. In: BICUDO, C. E. M.; MENEZES, N. A. Biodiversity in Brazil. São Paulo: CNPq, 1996. p. 289-295. 
MERRITT, R. W.; CUMMINS, K. W. An introduction to the aquatic insects of North America. Dubuque/Iowa: Kendall/Hunt publishing company, 1996. 862p.

NELSON, J. S. Fishes of the world. 3th.ed. New York: John Wiley \& Sons Inc, 2006. 600p.

OLIVEIRA, E. F.; GOULART, E. Distribuição espacial de peixes em ambientes lênticos: interação de fatores. Acta Scientiarum. Maringá, v.22, n.2, p. 445-453, 2000.

REIS, R. E., S. O. KULLANDER \& C. J. FERRARIS, JR. (eds.). 2003. Check list of the freshwater fishes of South and Central America. Editora da Pontifícia Universidade Católica do Rio Grande do Sul, Porto Alegre, Brasil.

SCHAEFER, S. A. Conflict and resolution: impact of new taxa on phylogenetic studies of the neotropical cascudinhos (Siluroidei: Loricariidae). In: MALABARBA, L. R. et al. Phylogeny and Classification of Neotropical Fishes. Porto Alegre: EDIPUCRS, 1998. p. 375-400.

TANIGUCHI, G. M.; BICUDO, D. C.; SENNA, P. A. C., 2004. Gradiente litorâneolimnético do fitoplâncton e ficoperifíton em uma lagoa da planície de inundação do rio MogiGuaçu. Revista Brasil. Bot., v.28, n.1, p. 137-147, jan./mar.2005.

TEIXEIRA, I.; BENNEMANN, S. T. Ecomorfologia refletindo a dieta dos peixes em um reservatório no sul do Brasil. Rev. Biota Neotropica, v.7, n.2, p. 67-76, 2007.

UIEDA, V. S.; CASTRO, R. M. C. Coleta e fixação de peixes de riachos. In: CARAMASHI, E. P.; MAZZONI, R.; PERES-NETO, P. R. (Orgs.). Ecologia de Peixes de Riachos. v.6., Rio de Janeiro: PPGE- UFRJ, 1999. p. 1-22. (Série Oecologia Brasiliensis).

UIEDA, V. S. Regime alimentar, distribuição espacial e temporal de peixes (Teleostei) em um riacho na região de Limeira-SP. 1983. 151p. Dissertação (Mestrado). Universidade Estadual de Campinas.

VADAS, R. L. The importance of omnivory and predatorregulation of prey in assemblages of North América. Env. Biol. Fish., v. 27, n.4, p. 85-302, 1990.

VARI, R. P.; MALABARBA, L. R. Neotropical Ichthyology: an overview. In: MALABARBA, L. R. et al. Phylogeny and Classification of Neotropical Fishes. Porto Alegre: EDIPUCRS, 1998. p. 1-11.

WIENS, J. A. On competition and variable environments. American Scientitist. New Haven, n.65, p. 590-597, 1987.

WINEMILLER, K. O.; JEPSEN, D. B. Effects of sazonality and fish movement on tropical river food webs. Journal of Fish Biology. London, n. 53 (A), p. 267-296, 1998.

ZAVALA-CAMIN, L. A. Introdução aos estudos sobre alimentação natural em peixes. Maringá: Eduem Nupelia, 1996. 129p. 